\title{
Instrumentation of broadband frequency domain thermoreflectance for measuring thermal conductivity accumulation functions
}

\author{
K. T. Regner, ${ }^{1}$ S. Majumdar, ${ }^{1}$ and J. A. Malen ${ }^{1,2, a)}$ \\ ${ }^{1}$ Department of Mechanical Engineering, Carnegie Mellon University, Pittsburgh, Pennsylvania 15213, USA \\ ${ }^{2}$ Department of Materials Science and Engineering, Carnegie Mellon University, Pittsburgh, \\ Pennsylvania 15213, USA
}

(Received 29 March 2013; accepted 15 May 2013; published online 6 June 2013)

\begin{abstract}
This paper describes the instrumentation for broadband frequency domain thermoreflectance (BBFDTR), a novel, continuous wave laser technique for measuring the thermal conductivity accumulation function. The thermal conductivity accumulation function describes cumulative contributions to the bulk thermal conductivity of a material from energy carriers with different mean free paths. It can be used to map reductions in thermal conductivity in nano-devices, which arise when the dimensions of the device are commensurate to the mean free path of energy carriers. BB-FDTR uses high frequency surface temperature modulation to generate non-diffusive phonon transport realized through a reduction in the perceived thermal conductivity. By controlling the modulation frequency it is possible to reconstruct the thermal conductivity accumulation function. A unique heterodyning technique is used to down-convert the signal, therein improving our signal to noise ratio and enabling results over a broader range of modulation frequencies $(200 \mathrm{kHz}-200 \mathrm{MHz})$ and hence mean free paths. @ 2013 AIP Publishing LLC. [http://dx.doi.org/10.1063/1.4808055]
\end{abstract}

\section{INTRODUCTION}

Recent advances in metrology have improved our understanding of thermal transport at the nanoscale, which has increasing importance as device sizes shrink and applications for nanotechnology become more prevalent. ${ }^{1-4}$ At these length scales, size effects in heat conduction are particularly relevant because the characteristic length scale of the material can be comparable to the mean free paths (MFPs) of energy carrying particles, including phonons and electrons. The reduction in thermal conductivity $(k)$ of the nanostructure is related to the thermal conductivity accumulation function $\left(k_{\text {accum }}\right)$, which describes cumulative contributions to bulk thermal conductivity from energy carriers with different MFPs ${ }^{5-11}$ Direct measurements of $k_{\text {accum }}$ can lead to enhanced understanding of nanoscale thermal transport.

Despite its importance to the fundamental thermal properties of matter, measurement of $k_{\text {accum }}$ was only first realized in $2007,{ }^{12}$ and has been more actively researched over the past three years. ${ }^{7-10,13}$ Innovative advancements focus on measuring $k_{\text {accum }}$ in semiconductors (i.e., phonon-dominated heat transport) as a function of a controllable experimental characteristic length scale $\left(L_{\mathrm{c}}\right)$, where only phonons with MFPs less than $L_{\mathrm{c}}$ contribute to the measured value of $k .{ }^{9,12,13}$ Most attempts utilize an experimental technique known as time domain thermoreflectance (TDTR). ${ }^{14-16}$ TDTR is a non-contact thermal conductivity measurement technique that uses ultrafast pulsed lasers. First, absorption of a pump pulse causes a temperature excursion at the sample surface. A probe pulse is time delayed with respect to the pump pulse using a mechanical delay stage. The amplitude of the reflected probe pulse is used to measure the temperature change at the sample sur-

\footnotetext{
a) Author to whom correspondence should be addressed. Electronic mail: jonmalen@andrew.cmu.edu
}

face based on thermoreflectance. A secondary modulation is induced on the train of pump pulses to enable measurement of the thermal response using a lock-in amplifier, thus increasing the signal to noise ratio.

One recent TDTR study observed reduced $k$ in Si for smaller laser spot sizes $\left(r_{\text {spot }}\right)$ and hypothesized that they were making direct measurements of $k_{\text {accum }}$ with $L_{\mathrm{c}}=2 r_{\text {spot }}{ }^{9}$ With this approach, however, the diffraction limit of light limits possible spot sizes and hence the range of the measurement. Alternatively, TDTR measurements by Koh and Cahill ${ }^{12}$ found that the thermal conductivity of semiconducting alloys depends on the secondary pump pulse modulation frequency, $f_{1}$. In this case, $L_{\mathrm{c}}$ is set by the thermal penetration depth $L_{\mathrm{p}}=\sqrt{k / \pi C f_{1}}$, where $C$ is the volumetric heat capacity. $L_{\mathrm{p}}$ identifies the depth normal to the surface at which the temperature amplitude is $e^{-1}$ of its surface amplitude. However, $L_{\mathrm{p}}$ in TDTR is restricted by an upper limit to the modulation frequency of $\sim 20 \mathrm{MHz}$ because at least four pulses are required in a modulation cycle and the pulse repetition rate of the common Ti-Sapphire oscillators is $80 \mathrm{MHz}$. Additionally, TDTR has several inherent time scales due to the use of a pulsed laser, including (i) the laser pulse width $(<0.5 \mathrm{ps})$, (ii) the delay time between pump and probe pulses ( $>100 \mathrm{ps})$, (iii) the time between laser pulses ( $\sim 10 \mathrm{~ns})$, and (iv) the secondary modulation frequency $(\sim 10 \mathrm{MHz})$. The multiple experimental time scales make it difficult to identify the most important. For example, Minnich et al. note that modulation frequency does not matter as much as the time delay, ${ }^{17}$ while experiments suggest otherwise. ${ }^{12}$ Alternatively, $L_{\mathrm{c}}$ can be set by the dimensions of nano-patterned heaters to extract the average phonon MFP of the substrate, ${ }^{13}$ but requires an optically transparent sample. Finally, $L_{\mathrm{c}}$ is proportional to the grating period in transient grating experiments, ${ }^{6-8,18}$ though this technique is as yet limited to thin, transparent membranes. 

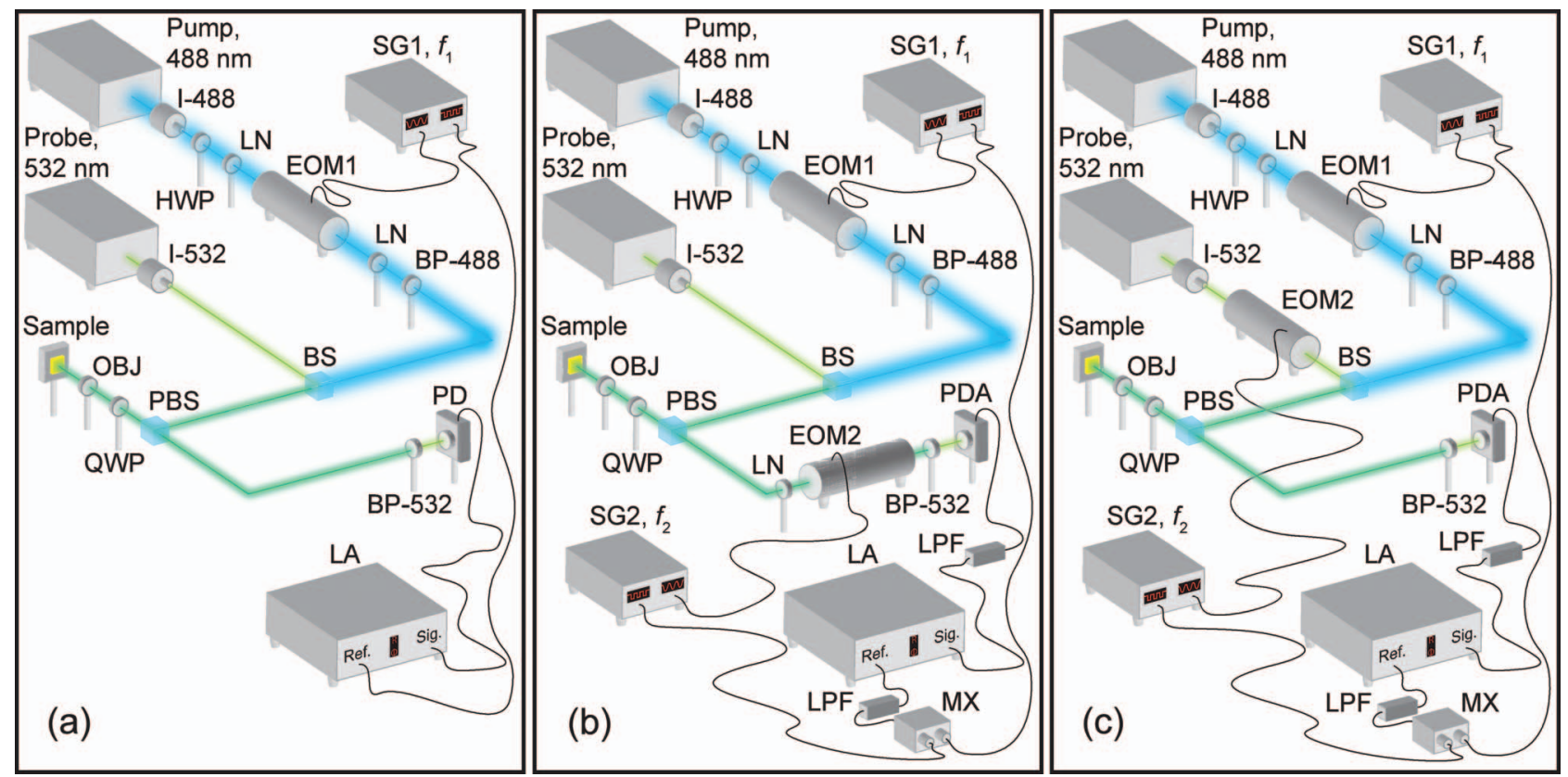

FIG. 1. (a) Schematic of traditional FDTR. (b) Schematic of BB-FDTR. The pump beam undergoes intensity modulation at frequency $f_{1}$ and gets focused on the sample. EOM2 induces an additional modulation in the reflected probe beam, heterodyning the thermal signal, allowing for higher heating frequencies at a lower measurement frequency, thereby increasing the measurement range of $k_{\text {accum. }}$. (c) Schematic of BB-FDTR with alternate location for EOM2. In this configuration, the heterodyning occurs at the sample surface but the thermal phase response cannot be isolated. Labels identifying the make and model of the components can be found in Table I.

In this work, we describe broadband frequency domain thermoreflectance (BB-FDTR), a MFP spectroscopy technique that uses high frequency modulation of continuous wave $(\mathrm{CW})$ lasers to vary $L_{\mathrm{p}}$ and measure an unprecedented range of $k_{\text {accum }}$ where $L_{\mathrm{c}}=L_{\mathrm{p}}$. This technique is based upon frequency domain thermoreflectance ${ }^{19,20}$ (FDTR) as shown in Figure 1(a), where a CW pump laser is intensity modulated at frequency $f_{1}$ and focused on to the sample surface. This periodic heat flux causes the surface temperature to change periodically at frequency $f_{1}$. The amplitude and phase of the surface temperature with respect to the incident heat flux contain information about the thermal properties of the sample, and are measured with a CW probe laser based on the thermoreflectance of the sample. The temperature response measured by the probe beam is fit to an analytical solution to the heat diffusion equation to determine the thermal conductivity of the sample.

To measure a large range of $k_{\text {accum }}$, it is necessary to create a thermal penetration depth in the material that is commensurate to the MFP of the phonons. This is achieved using modulation frequencies that limit the length scale of heat diffusion. Theoretically, since FDTR uses CW lasers, the pump can be modulated at infinite frequency to measure the entire $k_{\text {accum }}$. However, signal to noise ratios in FDTR decrease at larger heating frequencies due to the decreasing signal size and the presence of coherent noise and ambient noise at high frequency. Most early FDTR-based techniques do not modulate the pump laser higher than $2 \mathrm{MHz} ;{ }^{21-23}$ however, Pottier $^{24}$ and, more recently, Schmidt et al. ${ }^{19}$ and Ong et al. ${ }^{25}$ report heating frequencies up to $10 \mathrm{MHz}$ and $20 \mathrm{MHz}$, respectively.

Our BB-FDTR technique produces a high fidelity thermal signal by inducing an additional modulation on the re- flected, thermally modulated probe laser at frequency $f_{2}$. Heterodyning the probe laser allows for heating frequencies up to $200 \mathrm{MHz}$, but measurement of the thermal response at a much lower frequency, $f_{1}-f_{2}$, where the signal to noise ratio is much larger. Achieving this high frequency measurement is useful for accurately resolving interface thermal conductance, but more uniquely for measuring $k_{\text {accum }}$ over a broad range with minimal experimental modification (i.e., no changing focusing objectives or optical gratings, no special sample preparation, etc.). Since $L_{\mathrm{p}}$ and, hence, the MFPs accessible to BB-FDTR depend on thermal conductivity (through $\left.L_{\mathrm{p}}=\sqrt{k / \pi C f_{1}}\right)$, its range "adjusts" to the material, unlike spatially fixed cutoff length scales like $r_{\text {spot }}$ and the transient grating period. BB-FDTR was first introduced in our study of the phonon MFP spectra in crystalline and amorphous $\mathrm{Si}^{26}$ and we herein describe the instrumentation details, a similar alternative approach that was unsuccessful in our lab, and a detailed sensitivity analysis.

\section{EXPERIMENTAL SETUP}

The experimental setup for BB-FDTR is shown in Figure 1(b) with accompanying Table I that specifies the make and model of our key components. A $488 \mathrm{~nm}$, CW pump laser first travels through an optical isolator (I-488). The optical isolator prevents the back-reflected beam from entering the laser cavity, which causes instabilities in the laser output power. A half wave plate (HWP) is used to vertically polarize the light which is then focused into an electro-optic modulator (EOM1) that induces a sinusoidal intensity modulation at frequency $f_{1}$ on the transmitted vertically polarized light. High frequency EOMs typically have a small aperture to reduce the 
TABLE I. Component list for schematics in Fig. 1.

\begin{tabular}{lll}
\hline \hline Label & \multicolumn{1}{c}{ Component } & \multicolumn{1}{c}{ Model/description } \\
\hline I-532 & $532 \mathrm{~nm}$ isolator & Con-Optics M711A \\
I-488 & $488 \mathrm{~nm}$ isolator & Con-Optics M711C \\
BS & Beam splitter & Thor Labs BSF20-B \\
PBS & Polarizing beam splitter & Thor Labs PBS201 \\
QWP & Quarter wave plate & Thor Labs WPQ05M-532 \\
HWP & Half wave plate & Thor Labs WPH05M-488 \\
LN & Focusing lens & Thor Labs, varying focal lengths \\
BP-488 & 488 nm bandpass filter & Thor Labs FL-488-3 \\
BP-532 & 532 nm bandpass filter & Edmund Optics Techspec 65-155 \\
PDA & Amplified photodiode & Thor Labs PDA36A \\
PD & Photodiode & Thor Labs DET10A \\
EOM1/EOM2 & Electro-optic modulator & Con-Optics 350-80 \\
& 488 nm CW laser & Coherent Genesis MX \\
& 532 nm CW laser & Coherent Verdi G2 \\
OBJ & 20 $\times$ objective & Nikon CF Plan 20× ELWD \\
LA & Lock-in amplifier & SRS SR830 \\
SG1/SG2 & Signal generator & SRS SG384 \\
MX & Electronic mixer & MiniCircuits ZAD-3+ \\
LPF & Low pass filter & TTE LB3-120k-50-65B \\
\hline \hline
\end{tabular}

capacitance of the crystal, and as a result, we use a long focal length lens (LN) to focus into the EOMs. After exiting EOM1, the pump beam passes through a $488 \mathrm{~nm}$ bandpass filter (BP-488) and another lens to re-collimate the light. The $488 \mathrm{~nm}$ bandpass filter ensures that back-reflected probe light does not get directly modulated and detected in the signal after multiple reflections. A beam splitter (BS) transmits 53\% of the pump beam and is used to co-align the pump beam and probe beam along the same path.

The probe laser (532 nm, CW) passes through its own optical isolator (I-532) before encountering the beam splitter. The beam splitter is oriented such that $33 \%$ of the incoming probe beam continues towards the sample. The coaligned beams then pass through a polarizing beam splitter (PBS). The polarizing beam splitter allows the vertically polarized pump and probe beams to continue travelling towards the sample. A $532 \mathrm{~nm}$ quarter wave plate (QWP) then circularly polarizes the light and a $20 \times$ objective $(\mathrm{OBJ})$ is used to focus the lasers on to the sample surface. Based on the temperature change induced by the pump and the thermoreflectance of the transducer, a modulation is induced in the reflectivity of the surface and hence in the reflected probe at $f_{1}$.

When the pump and probe beams reflect from the sample surface, the circular polarization is reversed such that when the beams travel back through the quarter wave plate they become horizontally polarized. The horizontally polarized light is redirected towards the photodiode after passing back through the polarizing beam splitter. At this point, a lens is used to focus the light into EOM2. EOM2 induces an additional sinusoidal intensity modulation on the pump and probe beams at a frequency $f_{2}$. This heterodynes the pump and probe beams $\left(\sin \left(2 \pi f_{1} t\right) \times \sin \left(2 \pi f_{2} t\right)\right)$, creating frequency modulation components at $f_{1}-f_{2}$ and $f_{1}+f_{2}$. An optical bandpass filter (BP-532) is used to attenuate the pump beam and an amplified photodiode (PDA) converts the light into an electrical signal. The high frequency component of the signal $\left(f_{1}+f_{2}\right)$ is filtered out using a low pass filter (LPF) and a lock-in amplifier (LA) measures the amplitude $R$ and phase $\varphi$ of the signal at $f_{1}-f_{2}$. Heterodyning the signal allows for the use of an amplified photodiode; in FDTR, an amplified photodiode cannot be used due to low bandwidth.

The frequency $f_{1}-f_{2}$ is chosen such that it is less than $102 \mathrm{kHz}$. This allows for the use of an SR-830 lock-in amplifier (range of $1 \mathrm{mHz}$ to $102 \mathrm{kHz}$ ). The SR-830 lock-in amplifier has an advantage over the SR-844 lock-in in that it does not internally mix the signal with a square wave, which causes unwanted detection of the odd harmonics of the signal. We set $f_{1}$ and $f_{2}$ such that $f_{1}-f_{2}$ is maintained constant at $86 \mathrm{kHz}$ throughout the experiment. This, along with the low pass filter, ensures that higher harmonics do not contribute to the signal since they are out of the SR-830 range. This frequency, at the upper end of the SR-830 range, also minimizes $1 / f$ noise without exceeding the low-pass cutoff of our filter $\left(f_{\text {cutoff }}=120 \mathrm{kHz}\right)$.

To make a meaningful measurement at $f_{1}-f_{2}$, the lockin amplifier needs a reference signal at $f_{1}-f_{2}$. Since $f_{1}-f_{2}$ is not directly produced by either of the signal generators (SG) that drive the EOMs, it is generated using an electronic mixer (MX). The electronic mixer multiplies the input signals at $f_{1}$ and $f_{2}$ (from alternate outputs on signal generators 1 and 2) to create an output signal at $f_{1}+f_{2}$ and $f_{1}-f_{2}$. An identical low pass filter attenuates the high frequency component and the component at $f_{1}-f_{2}$ is used as the reference for the lock-in amplifier.

EOM1 and EOM2 need high voltage electrical signals to induce modulation in the incoming light. These signals are provided by signal generators 1 and 2 and amplified by the EOMs' amplifiers (not shown; Con-Optics M200). The shape of the voltage signal provided by the signal generator determines the shape of the light modulation. Signal generators 1 and 2 provide sinusoidal waveforms with frequency $f_{1}$ and $f_{2}$ and a peak-to-peak voltage of $0.2 \mathrm{~V}$. This voltage produces a large thermal signal without significantly increasing the internal temperature of the EOMs, which causes drift in the DC offset. The DC offsets of the EOM amplifiers are carefully tuned to provide the maximum AC signal without waveform distortion.

During the experiment, $f_{1}$ and $f_{2}$ are concurrently swept by locking the time base of signal generators 1 and 2 and fixing the frequency difference. The measurement frequencies are defined by a heating frequency vector, $\bar{f}_{1}$, that provides the frequency for signal generator 1 to drive EOM1. The driving frequency of EOM2 is determined such that $\bar{f}_{2}=\bar{f}_{1}+86 \mathrm{kHz}$. At each measurement point $\left(\bar{f}_{1}\right.$ is a 60 point vector uniformly spaced on a logarithmic axis between $200 \mathrm{kHz}$ and $200 \mathrm{MHz}$ ), the amplitude and phase with respect to the reference are recorded from the lock-in amplifier.

To determine the heating frequency dependent thermal conductivity of the sample the phase difference between the surface temperature and heat flux, $\varphi_{\text {thermal }}$, must be isolated. At any particular heating frequency, directly after the light reflects from the sample surface, the phase difference between the pump and the probe will be $\varphi_{\text {thermal }}$, i.e., if $\varphi_{\text {pump }}=0$, then $\varphi_{\text {probe }}=\varphi_{\text {thermal }}$. Both beams then travel through all the same 
optical components from the sample to the photodiode and both electrical signals encounter the same electrical components from the photodiode to the lock-in amplifier. Assuming the phase lag added to the signal from the optical components and photodiode is independent of the wavelength of the light, then $\varphi_{\text {pump }}=\varphi_{\text {opt/elec }}$ and $\varphi_{\text {probe }}=\varphi_{\text {thermal }}+\varphi_{\text {opt/elec }}$ at the input of the lock-in amplifier, where $\varphi_{\text {opt/elec }}$ is the phase lag added from the optical and electrical components. The lockin amplifier measures the phase with respect to the reference such that the measured phase differences $\varphi_{\text {pump,m }}=\varphi_{\text {optelec }}$ $-\varphi_{\text {ref }}$ and $\varphi_{\text {probe,m }}=\varphi_{\text {thermal }}+\varphi_{\text {opt/elec }}-\varphi_{\text {ref. }}$. Therefore, $\varphi_{\text {thermal }}$ can be isolated by subtracting the phase response from two experiments: one where the phase response of the probe is measured with a $532 \mathrm{~nm}$ bandpass filter installed before the photodiode and another where the phase response of the pump is measured with a $488 \mathrm{~nm}$ bandpass filter installed before the photodiode, i.e., $\varphi_{\text {thermal }}=\varphi_{\text {pump,m }}$ $-\varphi_{\text {probe,m }}$.

There are two options for the location of EOM2: (i) before the sample, such that the probe beam is modulated before co-aligning with the pump beam (Figure 1(c)) or (ii) after the sample, as in Figure 1(b). When EOM2 is placed before the sample, the probe beam is modulated at $f_{2}$ before incidence on the sample. Consequently, the heterodyning occurs at the sample surface, and only the probe beam is heterodyned (since only the pump is inducing a significant temperature change and only the probe has a high thermoreflectance). The phase difference measured in the probe is $\varphi_{\text {probe, } m}=\varphi_{\text {thermal }}$ $+\varphi_{\text {optelec }}-\varphi_{\text {ref }}$ and must be measured at $f_{1}-f_{2}$. To isolate $\varphi_{\text {thermal }}$ as described above, we run the experiment twice to obtain the phase difference in the pump and subtract the two measurements. When EOM2 is before the sample, however, the pump beam never gets heterodyned, i.e., there is no frequency component at $f_{1}-f_{2}$, and we cannot make a subtraction of $\varphi_{\text {probe,m }}$ and $\varphi_{\text {pump,m }}$ to isolate $\varphi_{\text {thermal }}$. While this, our initial configuration, resulted in similar amplitude response, the phase was not resolvable.

To highlight the effectiveness of the heterodyning technique in the high frequency regime, the normalized temperature response and phase response for c-Si at $T=311 \mathrm{~K}$ was measured with traditional FDTR and BB-FDTR setups from $f_{1}=200 \mathrm{kHz}$ to $200 \mathrm{MHz}$ and is shown in Figure 2. FDTR measurements were made with amplified and unamplified photodiodes. At low frequencies, the phase data measured using each setup is the same with low noise (see inset in Figure 2). However, at higher frequencies ( $>20 \mathrm{MHz}$ ), the signal to noise ratio in the traditional FDTR setup decreases drastically because as the frequency increases, the signal size decreases (see Sec. IV) but ambient and coherent noise increase. Coherent noise comes primarily from the high voltage signal carried by the cables connecting the EOM amplifier and EOM, which broadcasts at the same frequency as the thermal signal. It is picked up by the cable carrying the temperature response between the photodiode and lock-in amplifier, which acts as an antenna. BB-FDTR, however, increases the signal to noise ratio by completely eliminating coherent noise (signal is measured at $f_{1}-f_{2}$ while the EOMs are driven at $f_{1}$ and $f_{2}$ ) and reducing effects from ambient noise by measuring the signal at a much lower frequency, $f_{1}-f_{2}$,

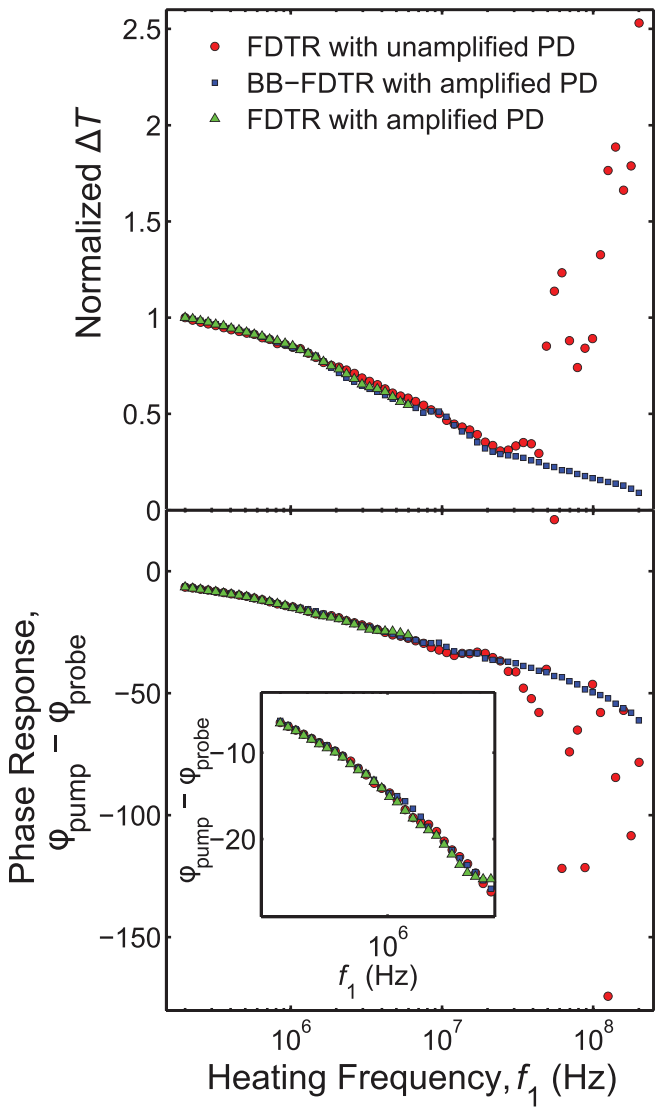

FIG. 2. Normalized temperature and phase response from traditional FDTR with an unamplified photodiode from $200 \mathrm{kHz}$ to $200 \mathrm{MHz}$ and an amplified photodiode up to the bandwidth of the photodiode and BB-FDTR experiments with an amplified photodiode for $\mathrm{c}-\mathrm{Si}$ at $T=311 \mathrm{~K}$. In the low frequency regime, the data from traditional FDTR and BB-FDTR is the same and indicates no effect from using different photodiodes. As the frequency increases $\left(f_{1}>20 \mathrm{MHz}\right)$, signal to noise ratios decrease in traditional FDTR. Heterodyning the signal allows for large signal to noise ratios up to a heating frequency of $200 \mathrm{MHz}$.

and by using an amplified photodiode since $f_{1}-f_{2}$ is within the bandwidth of the amplified photodiode. Traditional FDTR with the same amplified photodiode shows the same phase response as BB-FDTR up to the bandwidth $(<10 \mathrm{MHz})$. Consistency of all three data series below $10 \mathrm{MHz}$ validates the heterodyne approach and confirms that the response is not affected by the selection of photodiode. Thus, BB-FDTR yields a measurable signal for heating frequencies up to $200 \mathrm{MHz}$, an order of magnitude larger than traditional FDTR, and is only limited by the frequency restrictions of the EOMs. It is worth noting, however, that some noise is generated at $86 \mathrm{kHz}$ due to crosstalk between the EOM cables, and we have found that spatial separation of the EOM amplifiers is the most effective means of reduction.

\section{SAMPLE PREPARATION}

To increase the signal to noise ratio, samples are sputtered with a thin surface coating, known as a transducer layer. The transducer layer, usually a metal film, reduces optical penetration depth, eliminating volumetric heating in the sample, and is chosen to maximize the signal size by selection of a material with a highly temperature dependent 
reflectivity at the wavelength of the probe laser. The temperature dependent reflectivity arises from the coefficient of thermoreflectance of the transducer layer, $\beta_{532 \mathrm{~nm}}$, and is given by $\beta_{532 \mathrm{~nm}}=\left[1 / \rho_{0,532 \mathrm{~nm}}\right]\left[\mathrm{d} \rho_{532 \mathrm{~nm}}(T) / \mathrm{d} T\right]$, where $\rho_{532 \mathrm{~nm}}(T)$ is the temperature dependent reflectivity and $\rho_{0,532 \mathrm{~nm}}$ is the nominal reflectivity of the transducer at the probe wavelength at ambient temperature. Thus, the periodic change in reflectivity is $\rho_{532 \mathrm{~nm}}\left(T_{\omega}\right)=\beta_{532 \mathrm{~nm}} \rho_{0,532 \mathrm{~nm}} T_{\omega}$, where $T_{\omega}$ is the periodic temperature variation of the sample surface. ${ }^{20} T_{\omega}$ can be increased by increasing the intensity of the absorbed pump laser, which is accomplished by choosing a transducer material with high absorptivity at the pump laser wavelength $(488 \mathrm{~nm})$. For our system, Au is the ideal transducer material because it has high absorption at $488 \mathrm{~nm}$ and a high coefficient of thermoreflectance at $532 \mathrm{~nm} .{ }^{27}$

The interface thermal conductance, $G$, between the transducer layer and the sample is an unknown, important parameter in our experiment. To increase the sensitivity to $k_{\text {accum }}$ of the sample, $G$ must be as large as possible (see Sec. V). The Au transducer has a large mismatch in Debye temperature with $\mathrm{Si}$, and their interface has a relatively low $G$. Therefore, a $\mathrm{Cr}$ adhesion layer is used between the sample and the Au transducer to increase $G$ based on the similarity of Debye temperatures between $\mathrm{Cr}$ and $\mathrm{Si}$ compared to $\mathrm{Au}$ and $\operatorname{Si}\left(\theta_{\text {Debye,Cr }}=630 \mathrm{~K}, \theta_{\text {Debye,Si }}=645 \mathrm{~K}, \theta_{\text {Debye,Au }}=170 \mathrm{~K}\right)$. For results shown in this work, an $8 \mathrm{~nm} \mathrm{Cr}$ adhesion layer and a $56 \mathrm{~nm}$ Au transducer layer were deposited onto a single crystal, intrinsic silicon substrate $\left(\mathrm{c}-\mathrm{Si},<1 \times 10^{12} \mathrm{~cm}^{-3}\right.$ impurity atoms) using a Perkin Elmer $6 \mathrm{~J}$ sputtering system in the Carnegie Mellon Nanofabrication facility. Prior to the sputtering process, the native oxide layer on the c-Si substrate was removed with a buffered HF solution. Thicknesses of the $\mathrm{Cr}$ and $\mathrm{Au}$ layers were measured with X-ray reflectivity (XRR).

\section{ANALYTICAL SOLUTION}

The phase data is fit to a known analytical solution to the heat diffusion equation for the frequency domain temperature response of a multi-layered structure heated periodically with radial symmetry at the top surface..$^{28}$ It accounts for the Gaussian shape of the heat flux (pump) and calculates an average temperature response based on the Gaussian weighted sampling of the probe beam:

$$
\bar{T}=2 \pi Q_{\mathrm{o}} \int_{0}^{\infty} G(s) \exp \left(-\pi^{2} s^{2} 2 r_{\text {spot }}^{2}\right) s d s,
$$

where $Q_{\mathrm{o}}$ is the absorbed laser power, $r_{\mathrm{spot}}$ is the $1 / e^{2}$ laser spot radius, and $G(s)$ is the Hankel transform of the 1D, semiinfinite, periodic heat flux solution. For a single layer, $G(s)$ is given by Eq. (2):

$$
G(s)=\frac{1}{k\left(4 \pi^{2} s^{2}+i \omega / \alpha\right)^{1 / 2}},
$$

where $\omega$ is the angular heating frequency, $\alpha$ is the thermal diffusivity of the layer, and $s$ is the Hankel transform variable. $G(s)$ for a multilayered sample can be found in Ref. 28 for isotropic $k$ and for anisotropic $k$ by Schmidt et al. in Ref. 29. The phase response measured in BB-FDTR is fit to Eq. (1) to $k$ of the sample using a nonlinear regression until the mean square error is minimized.

In the high frequency limit, where $L_{\mathrm{p}} \ll r_{\text {spot }}$, the isotherms of the temperature profile will be relatively planar. The temperature response at the surface can be approximated with the 1D, planar heating solution with incident heat flux, $q(z=0)=q_{\mathrm{o}} \exp (i \omega t)$ :

$$
T_{\text {planar }}(z=0)=\frac{q_{\mathrm{o}}}{\sqrt{k C \omega}} \exp [i(\omega t-\pi / 4)] .
$$

In this case, the amplitude of the temperature response is inversely proportional to $\sqrt{k \omega}$ and the temperature response lags the heat flux by $\pi / 4$ radians in phase. For the two layered transducer/sample case, the temperature response is still inversely proportional to $\sqrt{\omega}$ but the phase is no longer $\pi / 4$.

In the experiments, the signal to noise ratio can be approximated by the amplitude of the temperature response, i.e., the larger the amplitude the larger the signal to noise ratio. Consequently, at lower heating frequencies, the signal to noise ratio is larger and increases with decreasing sample thermal conductivity. At high heating frequencies, the signal size decreases, and our heterodyne approach is critical.

The signal to noise ratio increases with increasing pump power, however, large pump powers can cause unwanted DC heating of the sample. Per Ref. 28, the DC temperature rise of the sample can be estimated by taking the low frequency limit of Eq. (1):

$$
\Delta T_{\mathrm{DC}}=\frac{Q_{\mathrm{o}}}{2 \sqrt{\pi} r_{\mathrm{spot}} k_{\mathrm{bulk}}},
$$

where $k_{\text {bulk }}$ is the bulk thermal conductivity of the sample. In BB-FDTR, $Q_{\mathrm{o}}$ was measured to be $3 \%$ of the total pump power and $5 \%$ of the total probe power. The DC temperature rise is accounted for by adding $\Delta T_{\mathrm{DC}}$ to the experimental temperature set point, which is controlled by a cryostat in our system.

\section{SENSITIVITY}

A sensitivity analysis was performed to examine how sensitive the solution to the diffusion equation is to different input parameters, $\beta$, over the entire frequency range. The model requires nominal values for the transducer layer thickness $\left(L_{\text {transducer }}\right)$, the transducer layer thermal conductivity $\left(k_{\text {transducer }}\right)$, the volumetric heat capacity of the transducer layer $\left(C_{\text {transducer }}\right)$, the thermal conductivity of the sample ( $\left.k_{\text {sample }}\right)$, the volumetric heat capacity of the sample $\left(C_{\text {sample }}\right)$, the interface conductance between the transducer layer and the sample $(G)$, and the laser spot radius $\left(r_{\text {spot }}\right)$. From Ref. 30, the sensitivity, $S_{\beta}$, is the logarithmic derivative of the phase response, $\varphi$, with respect to a change in one of the parameters, $\beta$, and is defined with Eq. (5):

$$
\begin{aligned}
S_{\beta} & =\frac{\mathrm{d} \ln (\varphi)}{\mathrm{d} \ln (\beta)} \\
\beta & =L_{\text {transducer }}, k_{\text {transducer }}, C_{\text {transducer }}, k_{\text {sample }}, C_{\text {sample }}, r_{\text {spot }}, G .
\end{aligned}
$$


TABLE II. Nominal values for fitting and the sensitivity analysis and the uncertainty ranges used to generate the shaded error regions in Fig. 5.

\begin{tabular}{lcc}
\hline \hline B & Nominal value & Uncertainty range \\
\hline$L_{\text {transducer }}$ & $64 \mathrm{~nm}$ & $\pm 5 \%$ \\
$C_{\text {transducer }}$ & $2470 \mathrm{~kJ} / \mathrm{m}^{3} \mathrm{~K}$ & $\mathrm{n} / \mathrm{a}$ \\
$k_{\text {transducer }}$ & $110 \mathrm{~W} / \mathrm{m} \mathrm{K}$ & $\pm 5 \%$ \\
$k_{\text {sample }}$ & $143 \mathrm{~W} / \mathrm{m} \mathrm{K}^{3} \mathrm{n} / \mathrm{a}$ \\
$C_{\text {sample }}$ & $1689 \mathrm{~kJ} / \mathrm{m}^{3} \mathrm{~K}$ & $\mathrm{n} / \mathrm{a}$ \\
$r_{\text {spot }}$ & $3.2 \mu \mathrm{m}$ & $\pm 0.1 \mu \mathrm{m}$ \\
$G$ & $210 \mathrm{MW} / \mathrm{m}^{2} \mathrm{~K}$ & $\pm 10 \mathrm{MW} / \mathrm{m}^{2} \mathrm{~K}$ \\
$\Delta \varphi_{\text {thermal }}$ & $\mathrm{n} / \mathrm{a}$ & $\pm 0.2^{\circ}$ \\
\hline \hline
\end{tabular}

The results of the sensitivity analysis about the nominal values (shown in Table II) are shown in Figure 3(a) for a $1 \%$ change in $\beta$. Figure 3(b) shows the sensitivity when $G=60 \mathrm{MW} / \mathrm{m}^{2} \mathrm{~K}$ and Figure 3(c) shows the sensitivity when $k_{\text {sample }}=10 \mathrm{~W} / \mathrm{m} \mathrm{K}$. A comparison of Figure 3(a) and Figure 3(b) shows that at higher heating frequencies the experiment is more sensitive to $k_{\text {sample }}$ when $G$ is larger. Hence, the $\mathrm{Cr}$ adhesion layer is used to increase $G$. A comparison of Figure 3(a) and Figure 3(c) shows lower overall sensitivity to $G$ but reduced sensitivity to $k_{\text {sample }}$ in samples with lower bulk thermal conductivity. While the heat capacity of the transducer and substrate, the thickness of the transducer, and the thermal conductivity of the transducer are moderately sensitive, they are well known values in our fitting. Additionally, the spot size value used in fitting $k_{\text {accum }}$ is most sensitive at lower heating frequencies, where $L_{\mathrm{p}} \sim r_{\text {spot. }}$. Notably, the heat capacity of the transducer makes the signal insensitive to

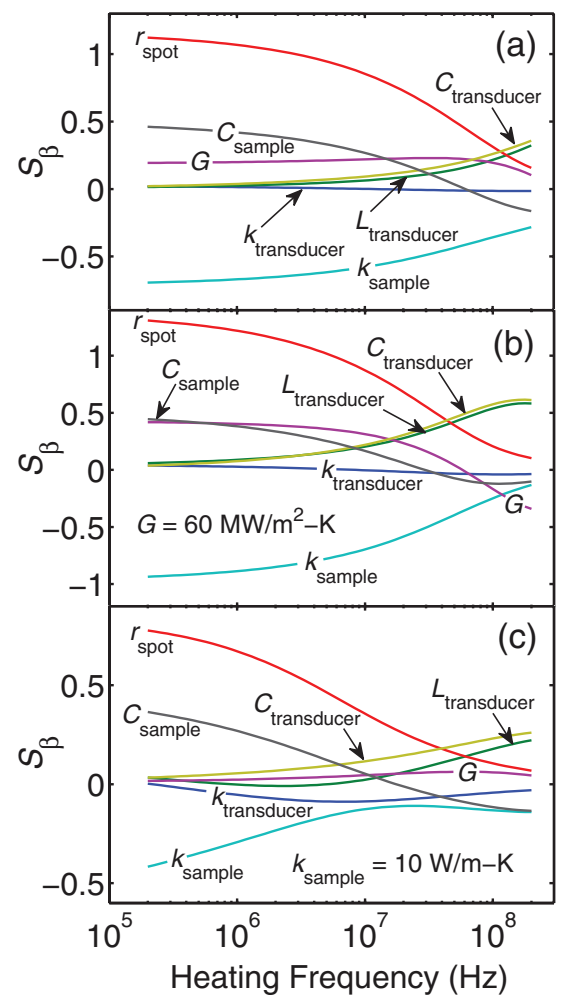

FIG. 3. (a) Sensitivity to a $1 \%$ change in nominal sample parameters, found in Table II, as defined by Eq. (5). The effect on the sensitivity from (b) decreased interface thermal conductance and (c) decreased sample thermal conductivity. $k_{\text {sample }}$ above $\sim 200 \mathrm{MHz}$, and future increases in the modulation frequency range need to be paired with thinner transducers to expand the measurement range of $k_{\text {accum }}$.

\section{SAMPLE RESULTS}

We present $k_{\text {accum }}$ here for an intrinsic c-Si sample at $T=311 \mathrm{~K}$ with nominal fitting values shown in Table II. $L_{\text {transducer }}$ was measured using XRR and $k_{\text {transducer }}$ was measured using a four-point resistivity measurement and the Wiedemann-Franz law. $C_{\text {transducer }}$ and $C_{\text {sample }}$ were assumed to be heating frequency independent and taken as the bulk value of $\mathrm{Au}$ and the bulk value of c-Si. This is because the long phonon MFPs probed by BB-FDTR contribute significantly to thermal conductivity but not to heat capacity $(<1 \%) .{ }^{5} k_{\text {bulk }}$ is from Ref. 31 and $r_{\text {spot }}$ was measured using the knife-edge profiling technique outlined in Ref. 26 . To determine the value of $G$, the last 15 points of the phase data were fit until mean square fitting error was minimized (as shown in the inset of Figure 4(a)) since $G$ is more sensitive in the high frequency regime (see Figure 3).

Fitting the phase data over the entire frequency range to a constant value of thermal conductivity yields a poor fit and underestimates the bulk value of Si by $34 \%$, as seen in Figure 4(a). Consequently, the phase data were divided into

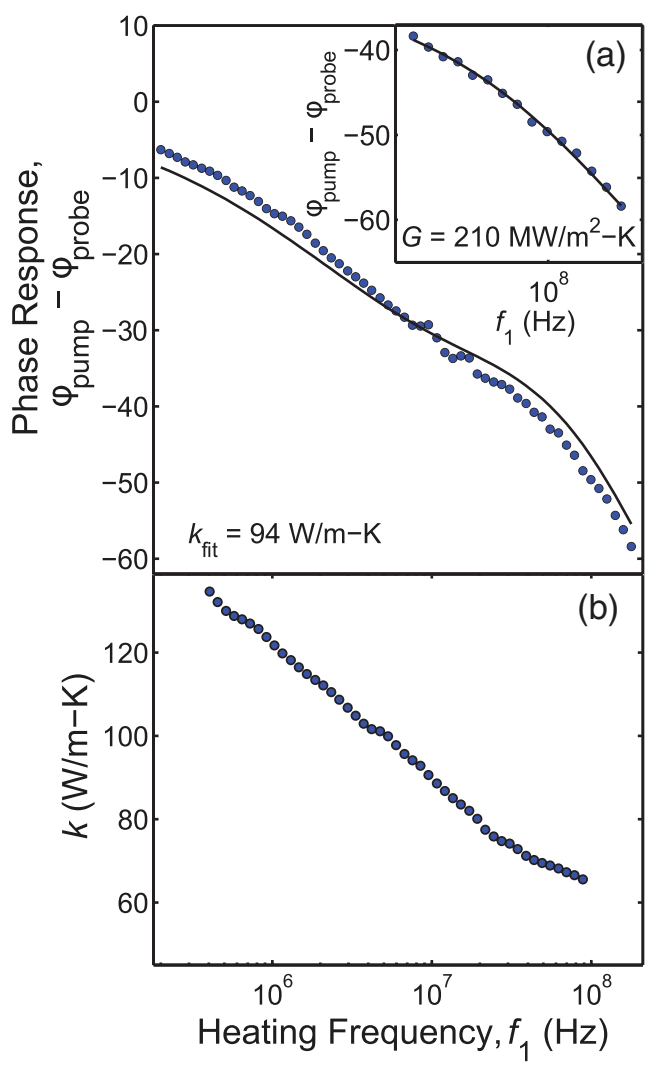

FIG. 4. (a) A constant $k$ fit to the phase response over the entire frequency range under predicts the bulk value of $\mathrm{Si}$ at $T=311 \mathrm{~K}$ and motivates a window fitting scheme. (b) Fitting $k$ in different windows of the phase response yields a heating frequency dependent thermal conductivity. The last 15 points of the phase data are fit to determine $G$ (inset), based on the sensitivity analysis. Minimizing mean square error yields a value of $G=210 \pm 10 \mathrm{MW} / \mathrm{m}^{2} \mathrm{~K}$. 


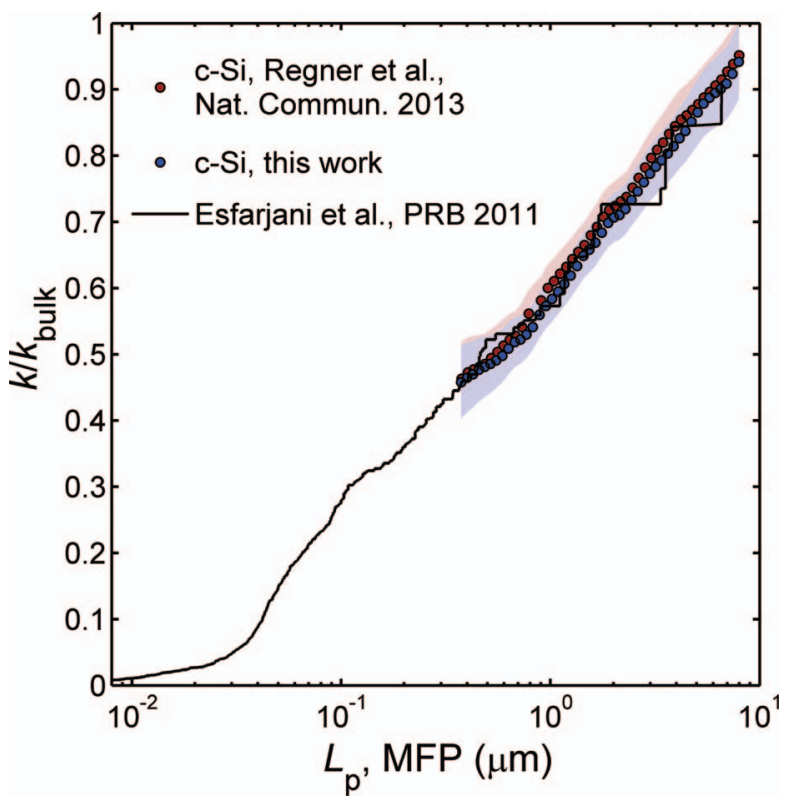

FIG. 5. Transforming the X-axis of Fig. 4(b) yields $k_{\text {accum }}$ for c-Si at $T=311 \mathrm{~K}$. The data is normalized to $k_{\text {bulk, }} \mathrm{Si}=143 \mathrm{~W} / \mathrm{m} \mathrm{K}^{31,32}$ and compare favorably with our prior results and numerical predictions from Ref. 9.

different windows and each window was fit individually. The frequency dependent thermal conductivity shown in Figure 4(b) was obtained by plotting the fitted value of $k$ from each window as a function of the median frequency of that fitting range. This plot is relatively independent of the window size as shown in the supporting information of Ref. 26. Transformation of the x-axis from frequency to $L_{\mathrm{c}}=L_{\mathrm{p}}$ yields $k_{\text {accum }}$, as seen for $\mathrm{Si}$ at $T=311 \mathrm{~K}$ in Figure 5. For simplicity, we have chosen $L_{\mathrm{c}}=L_{\mathrm{p}}$, though if a more rigorous proof, like that of Minnich for transient grating, ${ }^{6}$ suggests a more apt transformation (i.e., $L_{\mathrm{c}}$ is proportional to $L_{\mathrm{p}}$ ) the data can be rescaled.

Uncertainty in the measurements, $\Delta k$, is indicated as shaded regions in Figure 5 and was calculated as follows:

$$
\begin{aligned}
\Delta k & =\sqrt{\sum_{\beta}\left(\Delta k_{\beta}\right)^{2}}, \Delta k_{\beta}=\frac{\partial k_{\beta}}{\partial \beta} \Delta \beta, \\
\beta & =L_{\text {transducer }}, k_{\text {transducer }}, r_{\text {spot }}, G, \Delta \varphi_{\text {thermal }},
\end{aligned}
$$

where $\Delta k_{\beta}$ indicates the uncertainty from the selected modeling parameters $\beta$. The uncertainty in $k_{\text {accum }}$ due to $\Delta k_{\beta}$ depends on the sensitivity to that parameter $\partial k_{\beta} / \partial \beta$ and the uncertainty in that parameter $\Delta \beta$. Uncertainty ranges for the selected values of $\beta$ are shown in Table II. $\Delta \varphi_{\text {thermal }}$ considers a DC phase shift to the measured thermal phase response over all frequencies to examine the propagation of an error in the measured phase to $k_{\text {accum }}$ (see supporting information of Ref. 26). The uncertainty associated with $G$ was determined first by changing the other modeling parameters and fitting the high frequency phase data to minimize mean square error. The effective range of $G$ used for calculating $\Delta k$ is $\pm 10 \mathrm{MW} / \mathrm{m}^{2} \mathrm{~K}$. The resulting $k_{\text {accum }}$ is compared to numerical results from Ref. 9 and our original results for $\mathrm{c}-\mathrm{Si}^{26}{ }^{26}$ and shows favorable agreement. The temperature dependence of $k_{\text {accum }}$ for c-Si, as well as $k_{\text {accum }}$ for doped c-Si, a-Si, $\mathrm{SiO}_{2}$, and Pt can be found in Ref. 26.

\section{SUMMARY}

Here, we have presented the instrumentation for BBFDTR, a method for measuring the thermal conductivity accumulation function over an unprecedented range of MFPs. BB-FDTR uses a heterodyne technique which allows for high frequency heat flux modulation but measurement of the thermal signal at a much lower frequency, increasing the signal to noise ratio. $k_{\text {accum }}$ can be obtained by imposing $L_{\mathrm{c}}=L_{\mathrm{p}}$, such that the measured value of thermal conductivity only includes contributions from phonons with $\mathrm{MFP}<L_{\mathrm{p}}$. A window fitting scheme is used, where the unknown fitting variables are the thermal conductivity within that window and the interface thermal conductance between the transducer and the sample. The value of $G$ should be maximized to increase sensitivity to the substrate thermal conductivity and $k_{\text {accum. }}$. While BB-FDTR has as yet been applied to measure $k_{\text {accum }}$ from phonons, it may be possible to probe long MFP electrons in metals at low temperature.

\section{ACKNOWLEDGMENTS}

We acknowledge support from the National Science Foundation (NSF) CAREER Award (Award No. ENG1149374) and the ACS PRF DNI Award (Award No. PRF51423DN10).

${ }^{1}$ A. I. Hochbaum, R. Chen, R. D. Delgado, W. Liang, E. C. Garnett, M. Najarian, A. Majumdar, and P. Yang, Nature (London) 451, 163 (2008).

${ }^{2}$ B. Poudel, Q. Hao, Y. Ma, Y. Lan, A. J. Minnich, B. Yu, X. Yan, D. Wang, A. Muto, D. Vashaee, X. Chen, J. Liu, M. S. Dresselhaus, G. Chen, and Z. Ren, Science 320, 634 (2008).

${ }^{3}$ A. A. Balandin, Nat. Mater. 10, 569 (2011).

${ }^{4}$ R. J. Mehta, Y. L. Zhang, C. Karthik, B. Singh, R. W. Siegel, T. BorcaTasciuc, and G. Ramanath, Nat. Mater. 11, 233 (2012).

${ }^{5}$ K. Esfarjani and G. Chen, Phys. Rev. B 84, 085204 (2011).

${ }^{6}$ A. J. Minnich, Phys. Rev. Lett. 109, 205901 (2012).

${ }^{7}$ J. A. Johnson, A. A. Maznev, J. Cuffe, J. K. Eliason, A. J. Minnich, T. Kehoe, C. M. Sotomayor Torres, G. Chen, and K. A. Nelson, Phys. Rev, Lett. 110, 025901 (2013).

${ }^{8}$ J. A. Johnson, A. A. Maznev, J. K. Eliason, A. J. Minnich, K. Collins, G. Chen, J. Cuffe, T. Kehoe, C. M. Sotomayor Torres, and K. A. Nelson, Mater. Res. Soc. Symp. Proc. 1347, (2011).

${ }^{9}$ A. J. Minnich, J. A. Johnson, A. J. Schmidt, K. Esfarjani, M. S. Dresselhaus, K. A. Nelson, and G. Chen, Phys. Rev. Lett. 107, 095901 (2011).

${ }^{10}$ F. Yang and C. Dames, Phys. Rev. B 87, 035437 (2013).

${ }^{11} \mathrm{C}$. Dames and G. Chen, "Thermal conductivity of nanostructured thermoelectric materials," in Thermoelectrics Handbook: Macro to Nano, edited by D. M. Rowe (CRC Press, Boca Raton, 2006).

${ }^{12}$ Y. K. Koh and D. G. Cahill, Phys. Rev. B 76, 075207 (2007).

${ }^{13}$ M. E. Siemens, Q. Li, R. Yang, K. A. Nelson, E. H. Anderson, M. M. Murnane, and H. C. Kapteyn, Nat. Mater. 9, 26 (2009).

${ }^{14}$ C. A. Paddock and G. L. Eesley, J. Appl. Phys. 60, 285 (1986).

${ }^{15}$ W. S. Capinski and H. J. Maris, Rev. Sci. Instrum. 67, 2720 (1996).

${ }^{16}$ B. Bonello, B. Perrin, and C. Rossignol, J. Appl. Phys. 83, 3081 (1998).

${ }^{17}$ A. J. Minnich, G. Chen, S. Mansoor, and B. S. Yilbas, Phys. Rev. B 84, 235207 (2011).

${ }^{18}$ J. A. Johnson, A. A. Maznev, M. T. Bulsara, E. A. Fitzgerald, T. C. Harman, S. Calawa, C. J. Vineis, G. Turner, and K. A. Nelson, J. Appl. Phys. 111, 023503 (2012).

${ }^{19}$ J. A. Schmidt, R. Cheaito, and M. Chiesa, Rev. Sci. Instrum. 80, 094901 (2009). 
${ }^{20}$ J. A. Malen, K. Baheti, T. Tong, Y. Zhao, J. A. Hudgings, and A. Majumdar, J. Heat Transfer 133, 081601 (2011).

${ }^{21}$ N. Taketoshi, M. Ozawa, H. Ohta, and T. Baba, AIP Conf. Proc. 463, 315 (1999).

${ }^{22}$ K. Hatori, N. Taketoshi, T. Baba, and H. Ohta, Rev. Sci. Instrum. 76, 114901 (2005).

${ }^{23}$ F. Lepoutre, D. Balageas, P. Forge, S. Hirschi, J. L. Joulaud, D. Rochais, and F. C. Chen, J. Appl. Phys. 78, 2208 (1995).

${ }^{24}$ L. Pottier, Appl. Phys. Lett. 64, 1618 (1994).

${ }^{25}$ W. Ong, S. M. Rupich, D. V. Talapin, A. J. H. McGaughey, and J. A. Malen, Nat. Mater. 12, 410 (2013).
${ }^{26}$ K. T. Regner, D. P. Sellan, Z. Su, C. H. Amon, A. J. H. McGaughey, and J. A. Malen, Nat. Commun. 4, 1640 (2013).

${ }^{27}$ W. J. Scouler, Phys. Rev. Lett. 18, 445 (1967).

${ }^{28}$ D. G. Cahill, Rev. Sci. Instrum. 75, 5119 (2004).

${ }^{29}$ A. J. Schmidt, X. Chen, and G. Chen, Rev. Sci. Instrum. 79, 114902 (2008).

${ }^{30}$ R. M. Costescu, M. A. Wall, and D. G. Cahill, Phys. Rev. B 67, 054302 (2003).

${ }^{31}$ A. V. Inyushkin, A. N. Taldenkov, A. M. Gibin, A. V. Gusev, and H. J. Pohl, Phys. Status Solidi C 1, 2995 (2004).

${ }^{32}$ C. J. Glassbrenner and G. A. Slack, Phys. Rev. 134, A1058 (1964). 